\title{
Bladder squamous cell carcinoma in a pregnant woman: case report and review of the literature
}

Pablo A. Rojas ${ }^{1}$, Cristián González², Gonzalo P. Mendez ${ }^{3}$, Alejandro Majerson ${ }^{1}$ and Ignacio F. San Francisco ${ }^{\text {* }}$

\begin{abstract}
Background: Bladder tumors in pregnancy are extremely rare. No more than 50 cases have been published to date, including all histologic variants, and only three cases of bladder squamous cell carcinoma have been described.

Case presentation: We present a clinical case of a 31-year-old woman with bladder squamous cell carcinoma in the second trimester of pregnancy. After a C-section at 30 weeks, we performed radical cystectomy with extended bilateral lymphadenectomy, hysterectomy and right oophorectomy. The Studer neobladder technique was performed for urinary tract reconstruction. Definitive pathology showed invasive bladder squamous cell carcinoma, Grade 2, with microscopic infiltration of the perivesical fat, negative margins, and 3/28 lymph nodes with carcinoma (pT3aN2M0). The patient underwent 18 months of surveillance after radical cystectomy, without recurrence by PET-CT.

Conclusions: Bladder cancer in pregnant women is extremely rare but must be considered in those with recurrent gross hematuria and/or recurrent urinary tract infection. To our knowledge, this case involves the longest recurrencefree survival of a pregnant woman with squamous cell bladder cancer published thus far.
\end{abstract}

Keywords: Bladder cancer, Pregnancy, Radical cystectomy, Case report

\section{Background}

Bladder tumors inpregnancy are an extremely rare diagnosis. Currently, no more than 50 cases have been published, including all histologic variants [1].

\section{Case presentation}

We present the case of a healthy 31-year-old woman with an obstetric history of 1 pregnancy, 1 live birth, no abortions (G1P1A0), and a urinary tract infection in the first and second trimesters, without complications. At 21 weeks of gestation, the patient had gross hematuria. We performed cystoscopy and observed a lesion of $3-4 \mathrm{~cm}$ in the right wall not identifying the right ureteral

\footnotetext{
*Correspondence: isanfrancisco@med.puc.cl

${ }^{1}$ Departamento de Urología, Escuela de Medicina, Pontificia Universidad

Católica de Chile, Diagonal Paraguay 362, Santiago, Chile

Full list of author information is available at the end of the article
}

orifice (Fig. 1). Pelvic magnetic resonance imaging (MRI) without contrast was performed and showed a bladder tumor along the right wall that was $6.3 \times 3.5 \times 6 \mathrm{~cm}$ lateral to the right ureter and lymph nodes, $13 \times 9 \mathrm{~mm}$ in the right external iliac vessels and $10 \times 7 \mathrm{~mm}$ in the right internal iliac vessels (Fig. 2). The patient had no predisposing factors for bladder cancer.

From an obstetric perspective, the pregnancy was normal without complications.

At 26 weeks of gestation, we performed transurethral resection (TUR) of the bladder lesion. The biopsy showed well-differentiated, invasive bladder squamous cell carcinoma (pT2). Abdominal MRI and chest computed tomography $(\mathrm{CT})$ did not reveal metastatic disease. A staging study was performed after TUR to evaluate possible tumor dissemination and the need for systemic therapy. 


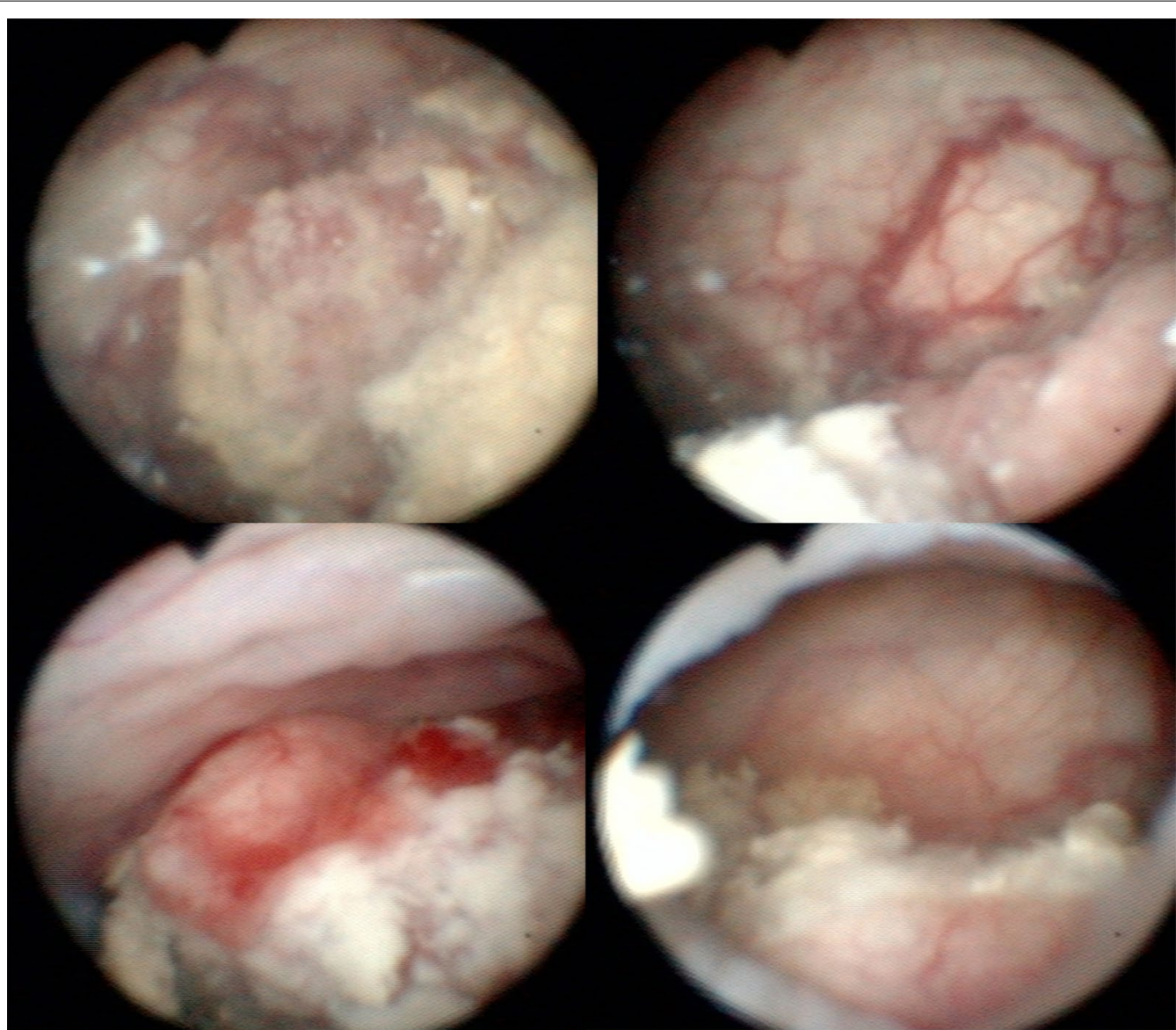

Fig. 1 Cystoscopy at 21 weeks of gestation

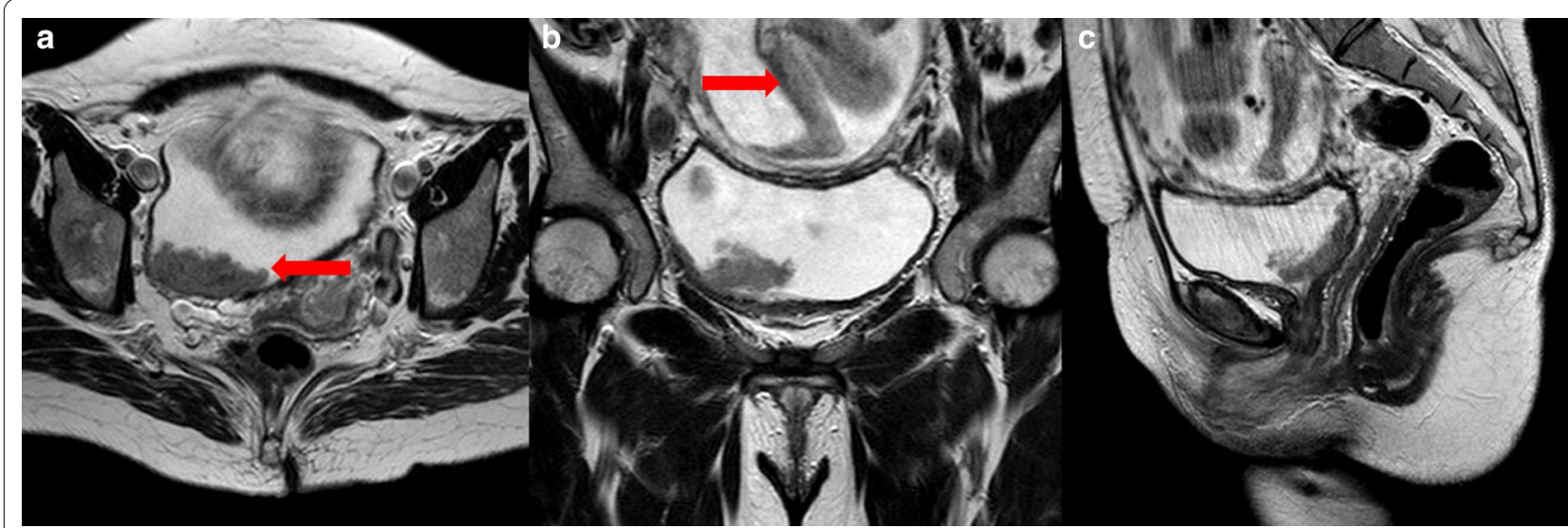

Fig. 2 MRI without gadolinium at 23 weeks of gestation. a Axial view of the bladder tumor (arrow). b Coronal view of the bladder tumor and fetus (arrow). c Sagittal view

The case was presented to a uro-oncology committee meeting and a maternal fetal medicine unit: the patient was offered the alternative of immediately terminating the pregnancy or waiting until 30 weeks for delivery and subsequently performing the surgery. The patient opted for the latter.

The patient underwent C-section at 30 weeks. The newborn was healthy, with no complications. 
As recommended by the multidisciplinary committee, the patient did not receive neoadjuvant chemotherapy based on the histology of pure squamous cell carcinoma. One month after delivery, we performed open radical cystectomy with extended bilateral lymphadenectomy through the common iliac vessels and presacrum, a hysterectomy and a right oophorectomy. Blood loss was $1100 \mathrm{cc}$. There were no other complications from the surgery. The Studer neobladder technique with $60 \mathrm{~cm}$ of terminal ileum was performed for urinary tract reconstruction. Cystostomy was carried out, and a urethral catheter was placed for anastomosis.

The patient recovered satisfactorily and was discharged 5 days postoperatively. The ureteral stents and cystostomy were removed two weeks after surgery.

Definitive pathology revealed invasive bladder squamous cell carcinoma, Grade 2, with microscopic infiltration of the perivesical fat, negative margins, and 3/28 lymph nodes with carcinoma (pT3aN2M0, Fig. 3). Again, evaluation by a multidisciplinary committee decided not to apply adjuvant chemotherapy/immunotherapy or radiation based on the histology and the lack of residual macroscopic disease.

Twenty days after the surgery, the patient presented with abdominal pain and fever. CT with contrast revealed uroperitoneum with neobladder filtration. The patient underwent exploratory laparotomy, which revealed $1200 \mathrm{cc}$ of uroperitoneum, leakage through the cystostomy site, suture line of the ileum and ureterovesical anastomosis. The filtration sites were closed with PDS sutures, and a urethral catheter and drainage were again installed. Five days after the surgery, the patient developed abdominal pain, and an increase in drainage output was observed. Cystography showed new filtration
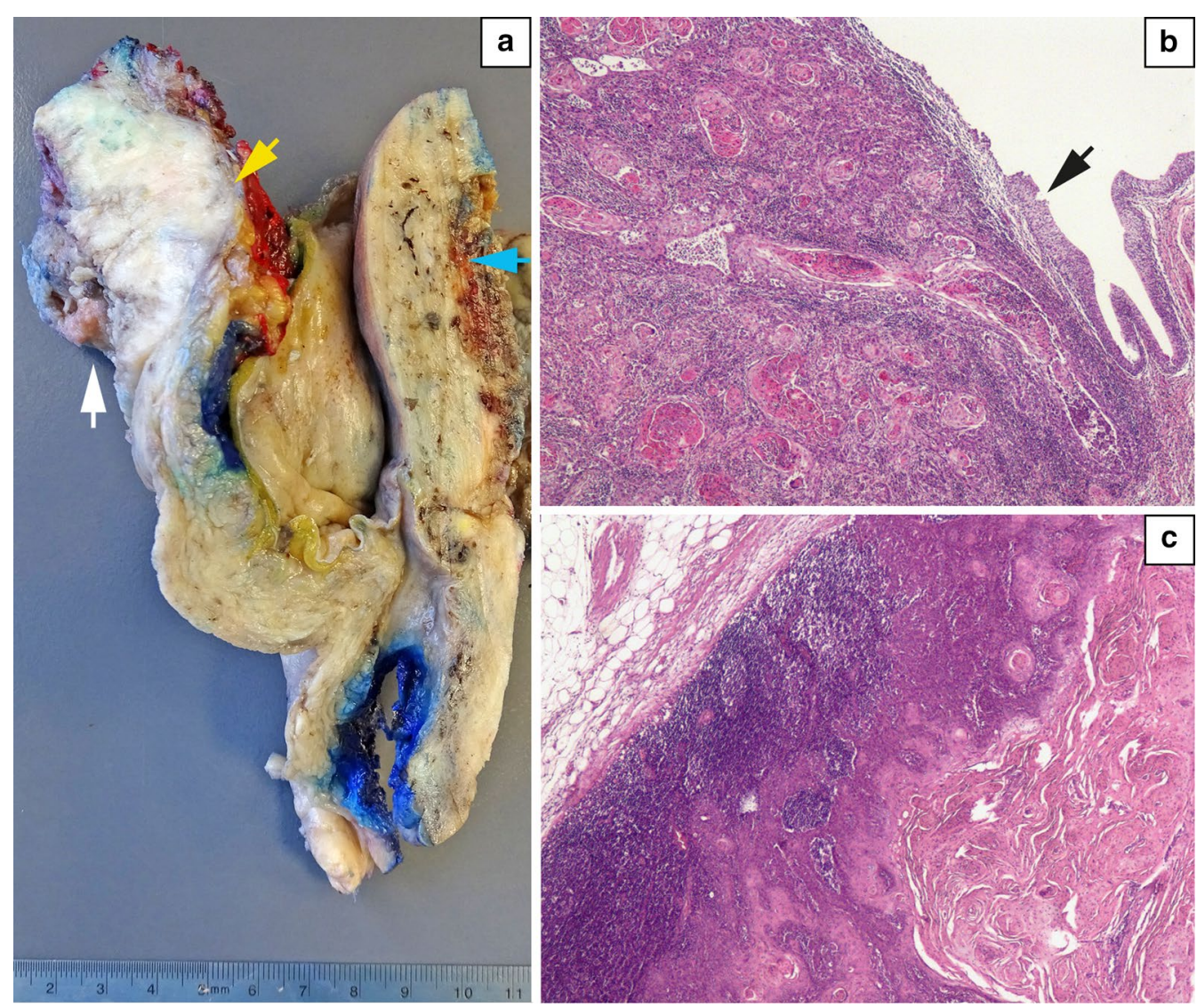

Fig. 3 a Longitudinal section of the formalin-fixed bladder and uterus. On the left, the bladder mucosa shows a bulky and exophytic, withe-gray tumor (white arrow) that invades through the complete bladder wall, reaching the perivesical adipose tissue (yellow arrow). On the right, the uterine wall and endometrium are thickened (blue arrow), with no evidence of tumor invasion. In between both walls, the vesico-uterine pouch can be recognized. b Microphotograph of the bladder mucosa showing a well-differentiated squamous cell carcinoma with multiple keratinizing epithelial groups (pink nests) invading the subepithelial connective tissue. The mucosa has epithelial denudation, and the residual urothelial lining is hyperplastic (arrow). (Hematoxylin and eosin stain, original magnification $\times 40$ ). c Image of one of the iliac lymph nodes with metastatic squamous cell carcinoma (hematoxylin and eosin stain, original magnification $\times 40$ ) 
in the anterior wall of the neobladder. The patient again underwent exploratory laparotomy, revealing leakage in two points of the suture line. The filtration sites were closed with PDS sutures. No drainage output or abdominal pain occurred but spontaneous diuresis was present. The patient was discharged at 3 weeks after the first reintervention.

At present, the patient has undergone 18 months of surveillance after radical cystectomy, without recurrence by follow-up PET-CT (Fig. 4), with complete continence, day and night, and no metabolic complications. Her baby is currently 19 months old and perfectly healthy.

\section{Discussion and conclusions}

Urological tumors in pregnant women are exceedingly rare, with an estimated incidence of $0.0013 \%$. The most frequent tumors are kidney tumors, followed by bladder tumors and finally pheochromocytomas [2].

Bladder tumors during pregnancy have been described since 1927, with transitional cell carcinoma being the most common (70\%) [3-7]. To our knowledge, only three cases of squamous cell bladder tumors have been reported in pregnant women (Table 1). Risk factors for squamous cell bladder tumors are a history of catheter use (clean intermittent self-catheterization or an indwelling catheter), smoking and recurrent urinary tract infections [8].

Among reported cases of squamous cell tumors, the women were in the third decade of life with pregnancies in the second trimester, similar to our patient. However, due to the aggressiveness of the disease, patients died within the first year of follow-up after cystectomy [7]. Therefore, to our knowledge, the patient we present in this report has the longest recurrence-free survival described in the literature.

Gross hematuria is frequent in pregnant women (up to $80 \%$ can present it), and the most common cause is cystitis [3,5]; considering the severity of the cases described above, cystoscopy is recommended for pregnant women with recurrent hematuria [7]. MRI can be used as a support tool for diagnosis and staging, and gadolinium can be used from the $23^{\text {rd }}$ week of gestation [2]. An abdomen and pelvis CT scan is not recommended in pregnant women because the fetus will be

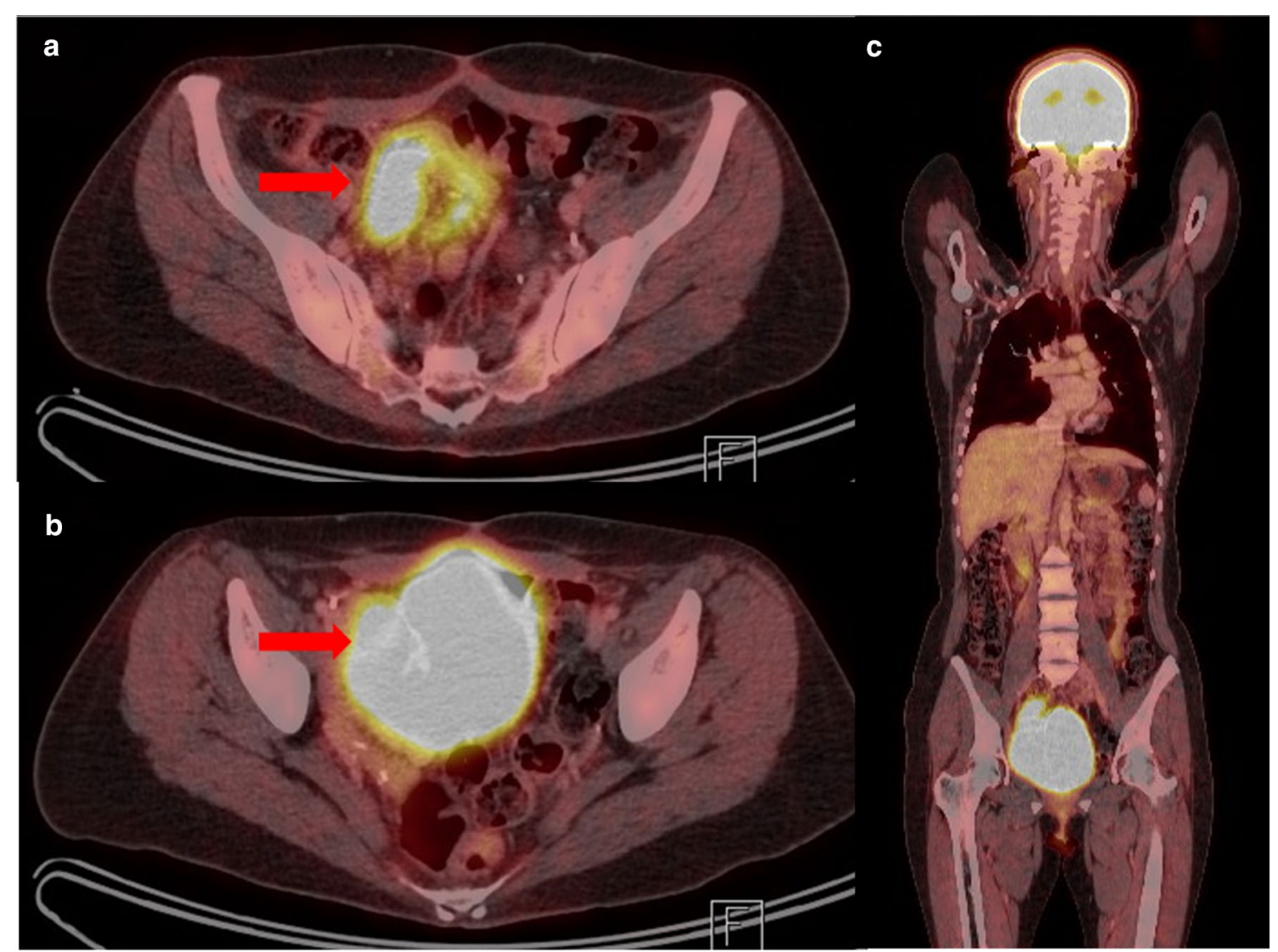

Fig. 4 PET-CT with F18-FDG. There was no evidence of locoregional recurrence or distant metastasis after 18 months of surveillance. a Axial view of chimney (arrow). b axial view of neobladder (arrow). c Coronal view 
Table 1 Bladder tumor in pregnant women. Prior studies

\begin{tabular}{|c|c|c|c|}
\hline Author & Publication's year & Number of patients & Histology \\
\hline Church [1] & 2013 & 1 patient, 27 years old & Squamous cell carcinoma \\
\hline Tyagi [3] & 2019 & 1 patient, 30 years old & Transitional cell carcinoma \\
\hline Mitra [4] & 2003 & 1 patient, 37 years old & Transitional cell carcinoma \\
\hline Spahn [6] & 2005 & 3 patients & $\begin{array}{l}2 \text { transitional cell carci- } \\
\text { noma, } 1 \text { squamous cell } \\
\text { carcinoma }\end{array}$ \\
\hline Alleemudder [7] & 2016 & 1 patient, 39 years old & Squamous cell carcinoma \\
\hline Shrotri [11] & 2008 & 1 patient, 36 years old & Transitional cell carcinoma \\
\hline
\end{tabular}

exposed to radiation above $50 \mathrm{mGy}$, though a chest CT scan is an alternative to staging because the radiation exposure of the fetus is minimal [9].

Once the diagnosis of the bladder tumor is established, it is essential to perform TUR, which is considered a low-risk procedure at any stage of pregnancy and only carries the usual risks associated with anesthesia [3]. Bipolar TUR may be safer [7]. Using histology, it is possible to categorize patients into risk groups to define management. Low-risk patients should be monitored with cystoscopy. In high-risk patients, a restaging TUR should be performed, and management should be decided according to the histology [2]. The safety of BCG instillations is currently unclear; however, there are reports of their use during the second trimester in a patient with carcinoma in situ [2]. Mitomycin is contraindicated as intravesical therapy during pregnancy due to its teratogenic effects [4].

When establishing that the tumor is infiltrating (pT2), the case must be staged. In addition, the gestation trimester must be considered. In the first and second trimesters, the pregnancy should be terminated, followed by cystectomy [4]; in the third trimester, the recommendation is to wait until 28-30 weeks of gestation and then perform cystectomy (after 3-4 weeks). These recommendations are based on the predicted percentage survival of the fetus, which is only $3 \%$ at 22 weeks of gestation but increases to $84 \%$ at 28 weeks [4].

Performing both surgeries at once is not recommended, as it would increase the risk of bleeding, considering pelvic congestion and the difficulty of performing a hysterectomy of a pregnant uterus [2]. Regarding urinary diversion, continent and noncontinent diversions have been described, without specific recommendations for the most appropriate surgical technique.

Currently, there is no evidence regarding adjuvant therapy with chemotherapy or systemic immunotherapy in pure squamous cell bladder tumors. Postoperative radiation therapy is an alternative to local control or in cases of positive margins [10].
In conclusion, bladder cancer in pregnant women is extremely rare and must be considered in women with recurrent gross hematuria and/or recurrent urinary tract infection. The management of these cases should be performed by a multidisciplinary team. The best time to perform radical cystectomy seems to be at least 4 weeks after $\mathrm{C}$-section and not at the same time. To our knowledge, this case constitutes the longest recurrence-free survival of a pregnant woman with squamous cell bladder cancer published in the literature.

\section{Abbreviations}

MRI: Magnetic resonance imaging;TUTR: Transurethral resection; CT: Computed tomography; PNLMP: Papillary neoplasms of low malignant potential.

\section{Authors' contributions}

PAR drafted and designed the article. CG reviewed the clinical case and current literature. GPM drafted and designed the article and contributed to the pathology analyses and images. AM performed the TURBT and reviewed the clinical case and current literature. IFS designed and reviewed the article, revising it critically for important intellectual content, performed radical cystectomy surgery and continued the patient's follow-ups. All authors have read and approved the manuscript.

Funding

This study has no funding.

Availability of data and materials Not applicable.

Ethics approval and consent to participate

This study was approved by the ethical committee of Hospital Clínico de la Pontificia Universidad Católica de Chile.

\section{Consent for publication}

Written informed consent was obtained from the case patient for publication of this report and any accompanying images. A copy of the written consent is available for review by the Editor of this journal.

\section{Competing interests}

The authors declare that they have no competing interests.

\section{Author details}

${ }_{1}^{1}$ Departamento de Urología, Escuela de Medicina, Pontificia Universidad Católica de Chile, Diagonal Paraguay 362, Santiago, Chile. ${ }^{2}$ División de

Ginecología y Obstetricia, Escuela de Medicina, Pontificia Universidad Católica de Chile, Santiago, Chile. ${ }^{3}$ Departamento de Anatomía-Patológica, Escuela de Medicina, Pontificia Universidad Católica de Chile, Santiago, Chile. 
Received: 21 July 2020 Accepted: 10 December 2020

Published online: 06 January 2021

\section{References}

1. Church E, Dieh A. A rare case of aggressive squamous cell carcinoma of the bladder in pregnancy. Obstet Med. 2013;6:182-3.

2. Khochikar MV. Management of urological cancers during pregnancy. Nat Rev Urol. 2010;7:195-205.

3. Tyagi S, Nelivigi G, Bhagat S. Management of bladder cancer in the second trimester of pregnancy. J Obstet Gynaecol India. 2019;69:20-3.

4. Mitra S, Williamson JG, Bullock KN, Arends M. Bladder cancer in pregnancy. J Obstet Gynaecol. 2003;23:440-2.

5. Muezzinoglu T, Inceboz U, Baytur Y, Nese N. Bladder carcinoma in pregnancy: unusual cause for frequent urinary tract infection-case report. Arch Gynecol Obstet. 2013;287:833-4.

6. Spahn M, Bader P, Westermann D, Echtle D, Frohneberg D. Bladder carcinoma during pregnancy. Urol Int. 2005;74:153-9.
7. Alleemudder DI, Alleemudder Al, Harry D, Fountain S. Bladder squamous cell carcinoma in pregnancy. J Obstet Gynaecol. 2016;36:388-9.

8. Manley KV, Hubbard R, Swallow D, Finch W, Wood SJ, Biers SM. Risk factors for development of primary bladder squamous cell carcinoma. Ann R Coll Surg Engl. 2017:99:155-60.

9. Yoon I, Slesinger TL. Radiation exposure in pregnancy. https://www.ncbi. nlm.nih.gov/books/NBK551690/. Accessed 2 Oct 2020.

10. Flaig TW, Spiess PE, Agarwal N, Bangs R, Boorjian SA, Buyyounouski MK, et al. Bladder Cancer, Version 3.2020, NCCN Clinical Practice Guidelines in Oncology. J Natl Compr Canc Netw. 2020;18:329-54.

11. Shrotri KN, Ross GC. Bladder carcinoma presenting during twin pregnancy. J Obstet Gynaecol. 2008;28:750-1.

\section{Publisher's Note}

Springer Nature remains neutral with regard to jurisdictional claims in published maps and institutional affiliations.
Ready to submit your research? Choose BMC and benefit from:

- fast, convenient online submission

- thorough peer review by experienced researchers in your field

- rapid publication on acceptance

- support for research data, including large and complex data types

- gold Open Access which fosters wider collaboration and increased citations

- maximum visibility for your research: over $100 \mathrm{M}$ website views per year

At BMC, research is always in progress.

Learn more biomedcentral.com/submissions 